\title{
Investigation of Shelf life of Potency and Activity of the Lactobacilli produced Bacteriocins through their exposure to various physicochemical stress factors
}

Dr. Myrto-Panagiota Zacharof ${ }^{1}$

Dr. Robert W. Lovitt ${ }^{2}$

${ }^{1}$ Multidisciplinary Nanotechnology Centre, Swansea University, Swansea, SA2 8PP, UK

${ }^{2}$ College of Engineering, Multidisciplinary Nanotechnology Centre, Swansea University, Swansea, SA2 8PP, UK

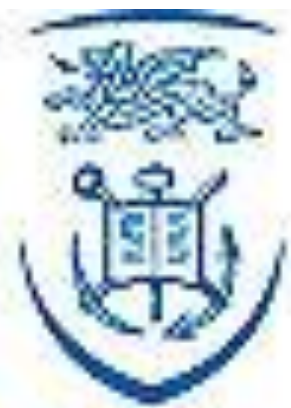

Swansea University Prifysgol Abertawe 


\section{Project Summary}

- Optimisation of physicochemical conditions of in vitro media for growth and bacteriocin production from Lactobacilli

- Development of a dose-response model for quantifying the bacteriocin by Lactobacilli amount produced based on the effect of Nisin (LAB commercially available bacteriocin) on the target strain

- Development of an extraction-concentration strategy of the bacteriocins majorly based on membrane filtration. 


\section{Project Summary}

- Testing the Activity and the Potency of the Commercially Available Nisin and the Produced Bacteriocins with Heat Treatment

- Testing the Activity and the Potency of the Commercially Available Nisin and the Produced Bacteriocins Through Storage effect on low temperatures

- Testing the Activity and the Potency of the Commercially Available Nisin and the Produced Bacteriocins through Dilution 


\section{Introduction}

- Lactobacilli: Rod shaped bacteria widely used in the food industry nowadays.

- They are mostly used in dairy industry due to their ability to ferment sugars especially glucose and galactose so to produce lactic acid .

- Research suggests that they produce antimicrobial activity metabolites

- These are active against other bacteria of the same genre and could be used as natural antimicrobials on their own or synergistically with other factors. 


\section{Introduction}

- Their production mechanism has been widely studied as well as their antimicrobial activity spectrum.

- Their antimicrobial nature is rather bacteriostatic than bactericidal as they mostly affect the cellular membrane of the target strains inactivating their reproduction mechanism.

- Nisin is the only commercially available bacteriocin. This emerges the need to further exploit these metabolites.

- The use of nisin as a natural additive to food, has been approved worldwide. 


\section{Introduction}

- Their production has several implications :

- They are extracellular metabolites of peptide nature so they are sensitive to conditions that may denaturise their peptide nature.

- If produced in large scale, using traditional fermentation techniques it might not be feasible to be extracted immediately from the fermented broths. Factors such as mechanical stress, dilution rate of the nutrient media, agitation or aeration might affect the bacteriocins quality.

- They were growth related metabolites so they are influenced from factors that negatively affect microbial growth (end product inhibition due to lactate excess, glucose exhaustion due to fastidious growth, microbial contamination).

- Their extraction becomes difficult as they may form micelles or clumps with the nitrogen sources already in the production medium. 


\section{Aims and Objectives}

- In this project, the previously referred problems were taken into consideration and a suitable strategy was developed.

- Three known bacteriocin producing strains were selected.

- A common optimised growth medium was be developed including optimum physicochemical conditions. 


\section{Aims and Objectives}

- The quantification method included a carefully selected target strain and a growth medium that ensured optimum growth of the target strain was developed.

- The self life of potency and activity was evaluated by exposing the extracted-concentrated bacteriocins and the crude extracts into several physicochemical stress factors. 


\section{Materials and Methods}

- Chemicals supplied by Sigma Aldrich UK, Fischer UK, Fluka UK.

- Lactobacillus casei NCIMB 11970 Lactobacillus plantarum NCIMB 8014, Lactobacillus lactis NCIMB 8586 and the target strain Lactobacillus delbruckii subsp.lactis 8117 were provided in a lyophilised form by National Collection of Food and Marine bacteria (NCIMB), Ferguson Building, Craibstone Estate, Aberdeen, Scotland, AB21 QYA.

- Pyrex glass tubes sealed with butyl rubber stoppers and alumina seals were used to measure the growth rate. The microbial growth was measured on UV/Vis Spectrometer Philips 8250 and the numerical results are calculated on MS Excel software 2003.

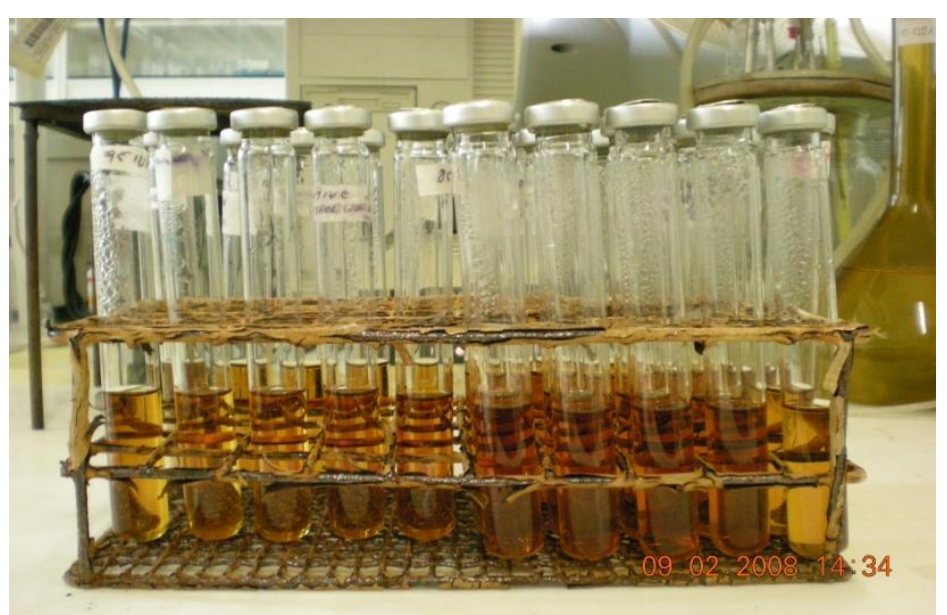

Fig 1. Test tubes containing growth medium

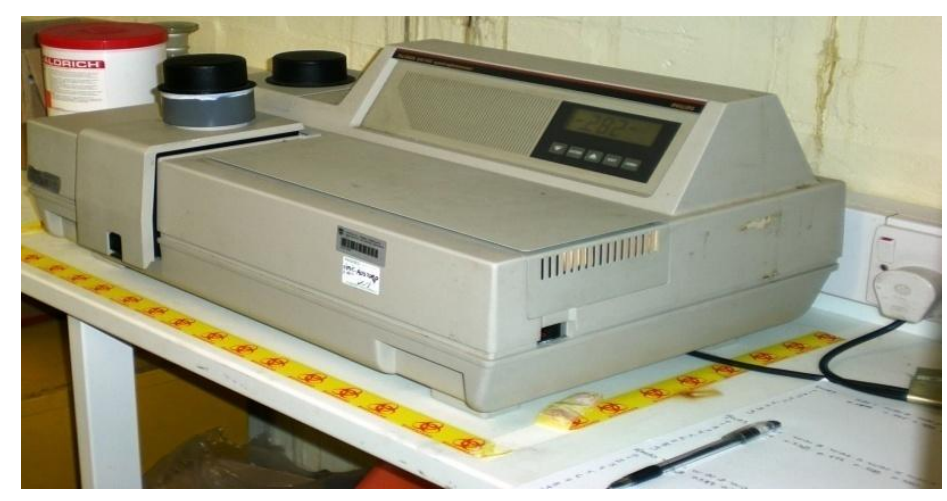

Fig 2.UV/Vis Philips 8250 Spectrophotometer 


\section{Materials and Methods}

- The amount of bacteriocins produced was measured by analysing the protein content using the Lowry method.

- Bacteriocins were sized using Dynamic Light Scattering technology.

- In order to quantify the amount of bacteriocins produed a liquid turbidometric assay based on nisin was developed

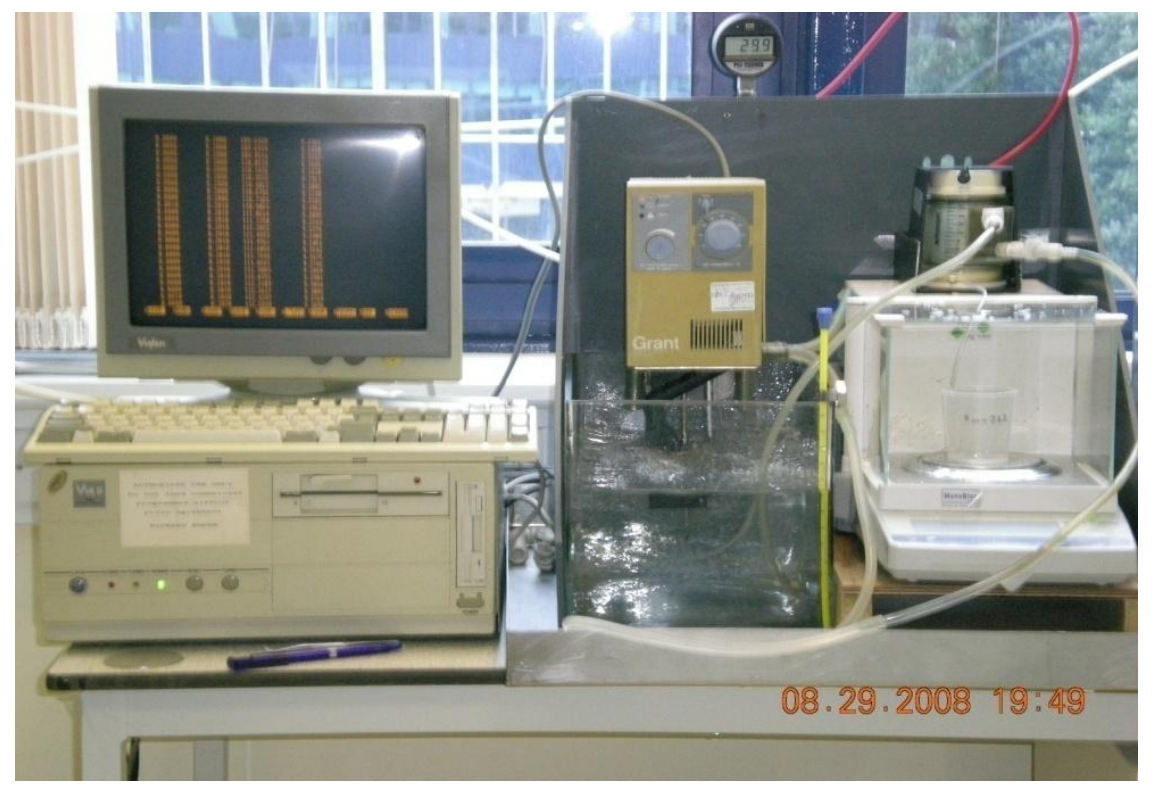

- Their activity and potency was tested against the target strain L.delbruckii

- To concentrate and purify the produced bacteriocins a serie of membrane filters of $30 \mathrm{kDa}$ (Cellulose acetate), $4 \mathrm{kDa}$ and $1 \mathrm{kDa}$ (Polysulfone) MWCO were used. The filters were purchased by Millipore, Microdyn-Nadir and Osmonics and fitted in Amicon cell 8200 unit reactor

Fig 3. Amicon Cell Unit Reactor 8200 System

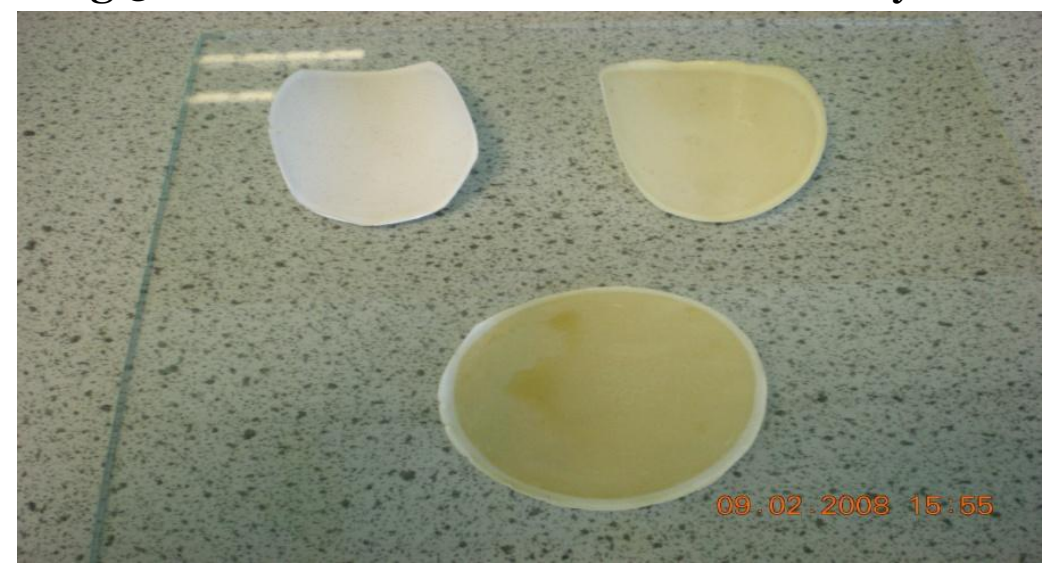

Fig 4. Ultrafiltration membrane filters (30kDa, $4 \mathrm{kDa}, 1 \mathrm{kDa}$ counter clockwise) 


\section{Results \\ Growth Media Development and Optimisation}

\begin{tabular}{|c|c|c|}
\hline 1. & Glucose $\left(\mathrm{C}_{6} \mathrm{H}_{12} \mathrm{O}_{6}\right)$ & $2 \% \mathrm{w} / \mathrm{v}$ \\
\hline 2. & Yeast Extract (Y.E.) & $1.5 \% \mathrm{w} / \mathrm{v}$ \\
\hline 3. & Bacteriological Peptone & $1 \% \mathrm{w} / \mathrm{v}$ \\
\hline 4. & Sodium acetate $\left(\mathrm{CH}_{3} \mathrm{COONa}\right)$ & $0.5 \% \mathrm{w} / \mathrm{v}$ \\
\hline 5. & Tri-Sodium citrate $\left(\mathrm{C}_{6} \mathrm{H}_{5} \mathrm{Na}_{3} \mathrm{O}_{7}\right)$ & $0.2 \% \mathrm{w} / \mathrm{v}$ \\
\hline 6. & Potassium Hydrogen Phosphate $\left(\mathrm{KH}_{2} \mathrm{PO}_{4}\right)$ & $0.2 \% \mathrm{w} / \mathrm{v}$ \\
\hline 7. & Magnesium Sulphate $\left(\mathrm{MgSO}_{4} 7 \mathrm{H}_{2} \mathrm{O}\right)$ & $0.02 \% \mathrm{w} / \mathrm{v}$ \\
\hline 8. & Manganese Sulphate $\left(\mathrm{MnSO}_{4} 3 \mathrm{H}_{2} \mathrm{O}\right)$ & $0.005 \% \mathrm{w} / \mathrm{v}$ \\
\hline 9. & 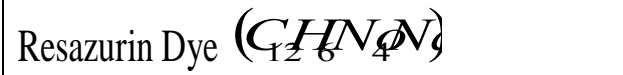 & $0.005 \% \mathrm{v} / \mathrm{v}$ \\
\hline
\end{tabular}

\begin{tabular}{|c|c|c|}
\hline 1. & Glucose $\left(\mathrm{C}_{6} \mathrm{H}_{12} \mathrm{O}_{6}\right)$ & $2 \% \mathrm{~W} / \mathrm{v}$ \\
\hline 2. & Yeast Extract(Y.E.) & $2 \% \mathrm{~W} / \mathrm{v}$ \\
\hline 3. & Sodium acetate $\left(\mathrm{CH}_{3} \mathrm{COONa}\right)$ & $19 \mathrm{w} / \mathrm{v}$ \\
\hline 4. & Tri-Sodium citrate $\left(\mathrm{C}_{6} \mathrm{H}_{5} \mathrm{Na}_{3} \mathrm{O}_{7}\right)$ & $1 \% \mathrm{w} / \mathrm{v}$ \\
\hline 5. & Potassium Hydrogen Phosphate $(\mathrm{KH}$ & $0.5 \% \mathrm{w} / \mathrm{V}$ \\
\hline 6. & Resazurin Dye $\overline{(C I H N \Phi)}$ & $0.005 \% \mathrm{v} / \mathrm{v}$ \\
\hline
\end{tabular}




\section{Results \\ Growth Media Development and Optimisation}

\begin{tabular}{|l|l|l|l|l|}
\hline \multirow{2}{*}{ Bacteria } & Media & $\begin{array}{l}\text { Maximum specific } \\
\text { Growth rate }(\mu \mathrm{max}, \\
\mathrm{h}-1)\end{array}$ & $\begin{array}{l}\text { Doubling time } \\
(\mathrm{t} d \mathrm{~h}, \mathrm{~h})\end{array}$ & $\begin{array}{l}\text { Final Biomass } \\
(\mathrm{g} / \mathrm{L})\end{array}$ \\
\hline \multirow{2}{*}{ L.casei } & $\begin{array}{l}\text { MRS modified } \\
\text { medium }\end{array}$ & 0.16 & 4.31 & 1.19 \\
\cline { 2 - 5 } & Optimised Medium & 0.24 & 2.87 & 2.43 \\
\hline \multirow{2}{*}{ L.plantarum } & $\begin{array}{l}\text { MRS modified } \\
\text { medium }\end{array}$ & 0.13 & 5.30 & 1.32 \\
\cline { 2 - 5 } & Optimised Medium & 0.30 & 2.30 & 2.63 \\
\hline \multirow{2}{*}{ L.lactis } & $\begin{array}{l}\text { MRS modified } \\
\text { medium }\end{array}$ & 0.07 & 9.85 & 0.69 \\
\cline { 2 - 5 } & Optimised Medium & 0.22 & 3.13 & 1.81 \\
\hline
\end{tabular}

Table 3: Comparative table of performance Optimised Medium and MRS modified medium 


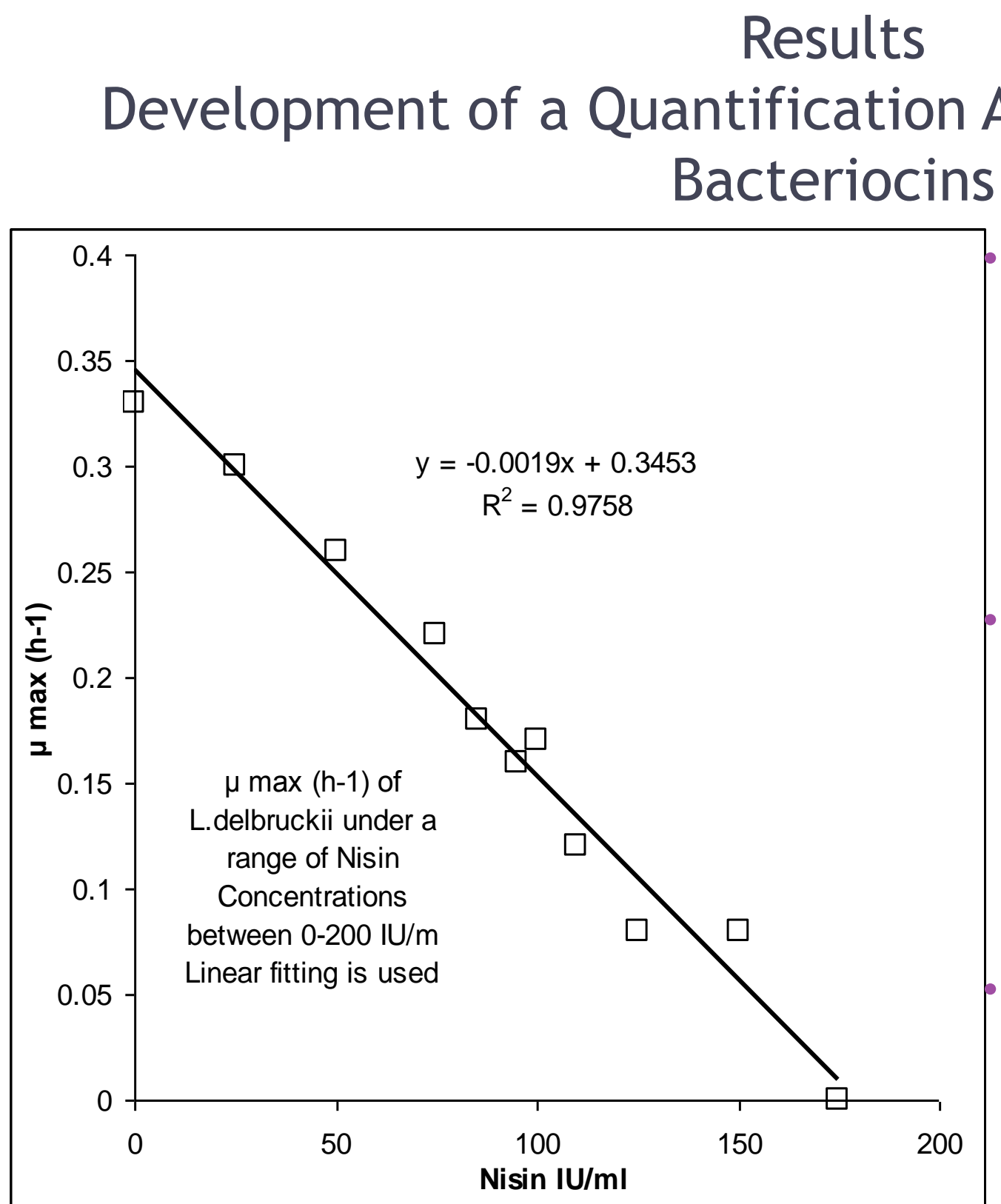

Fig 5. Standard curve of quantification of nisin assay
The assay was developed based on the growth rates of the target strain L.delbruckii occurring under different concentrations of nisin induced in the medium.

The assay had a limit value of 200 $\mathrm{IU} / \mathrm{ml}$ of nisin or of nisin-like activity antimicrobial compound. Beyond this value no growth occurs.

The Lactobacilli were tested for bacteriocin production both through measurement of protein content and through testing the supernatant for antimicrobial activity. 


\section{Results \\ Bacteriocin production by the selected Lactobacilli \\ Crude extracts and concentrated bacteriocins}

\begin{tabular}{|c|c|c|c|c|c|c|c|}
\hline Lactobacilli & $\begin{array}{l}\text { Growth rate } \\
\left(\mu, h^{-1}\right)\end{array}$ & $\begin{array}{l}\text { Doubling } \\
\text { Time (td,h) }\end{array}$ & $\begin{array}{l}\text { Amount of } \\
\text { bacteriocins } \\
\text { produced } \\
(\mathrm{IU} / \mathrm{ml})\end{array}$ & Lactobacilli & $\begin{array}{l}\text { Growth rate } \\
\left(\mu, h^{-1}\right)\end{array}$ & $\begin{array}{l}\text { Doubling } \\
\text { Time (td,h) }\end{array}$ & $\begin{array}{l}\text { Amount of } \\
\text { bacteriocins } \\
\text { produced } \\
(\mathrm{IU} / \mathrm{ml})\end{array}$ \\
\hline L.casei & 0.16 & 4.31 & 95 & L.casei & 0.12 & 5.75 & 115 \\
\hline L.plantarum & 0.16 & 4.31 & 95 & L.plantarum & 0.10 & 6.90 & 125 \\
\hline L.lactis & 0.14 & 4.92 & 105 & L.lactis & 0.09 & 7.66 & 130 \\
\hline
\end{tabular}




\section{Results \\ Potency of Bacteriocins and its Resistance to Deactivating Factors}

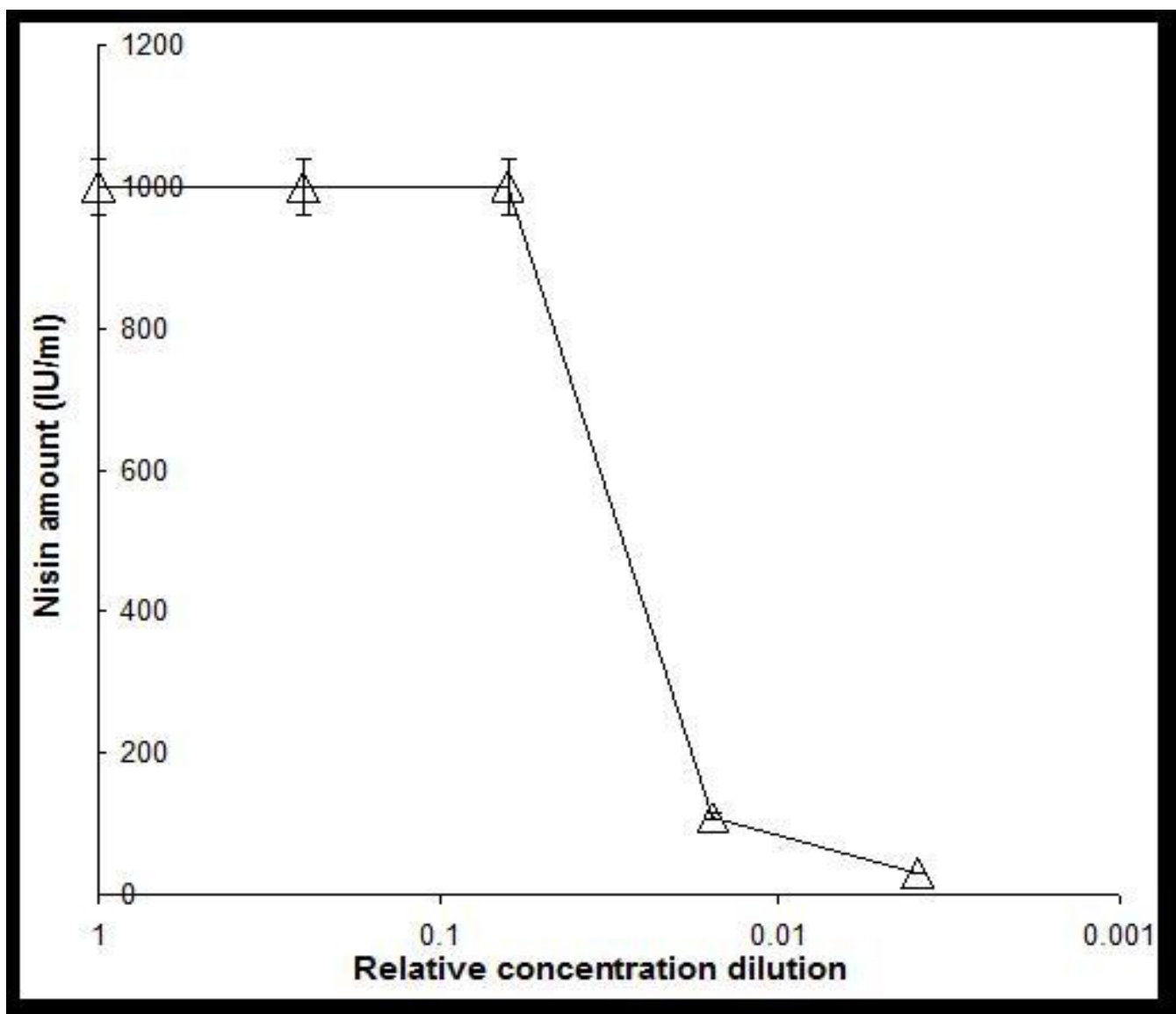

Figure 6 Effect of relative concentration dilution on the potency of nisin solution 1000 $\mathbf{I U} / \mathbf{m l}$ 


\section{Results \\ Potency of Bacteriocins and its Resistance to Deactivating Factors}

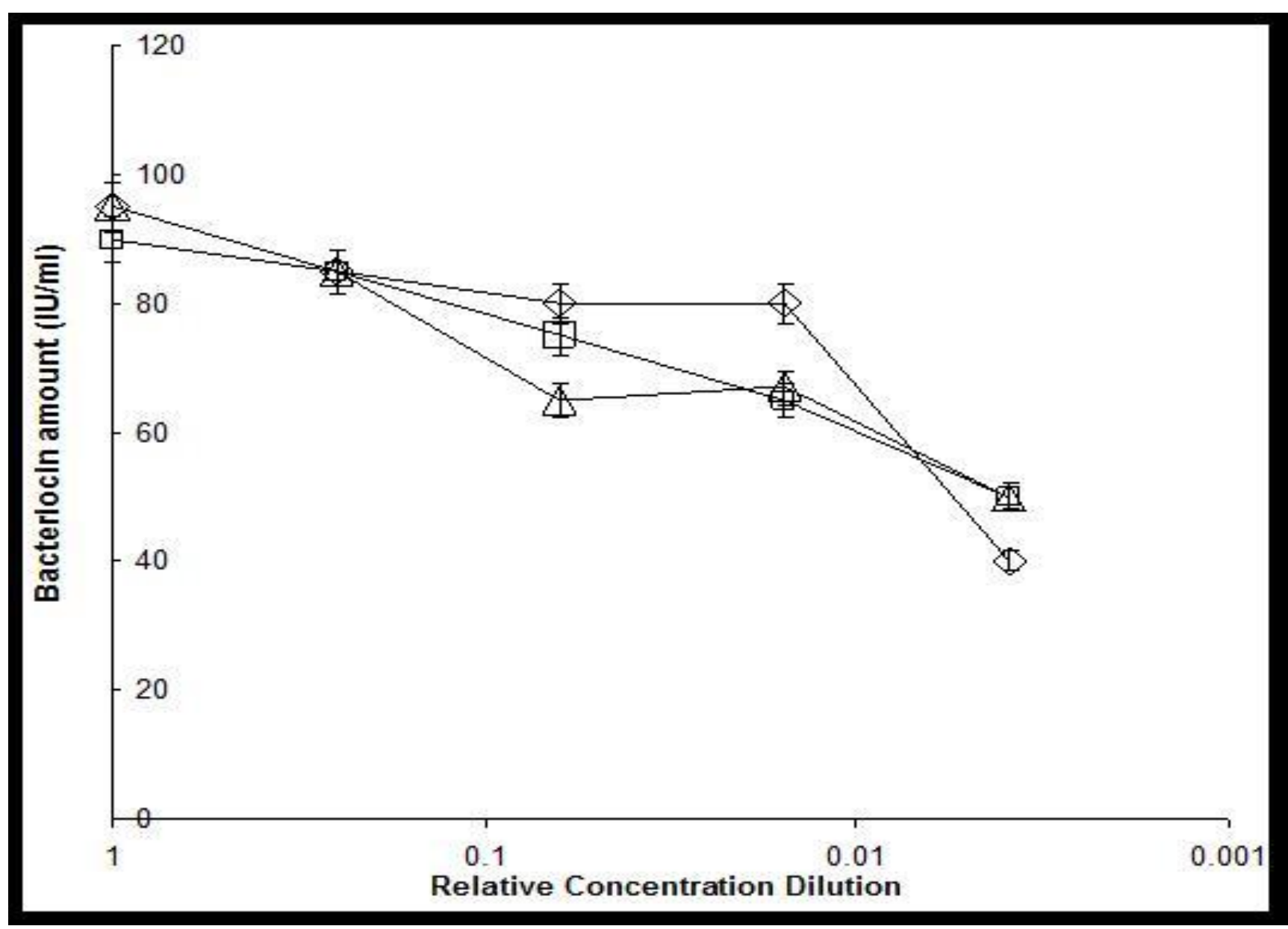

Fig 7. Potency of L.casei $(\diamond)$ L.plantarum $(\square)$ \&L.lactis $(\Delta)$ produced Bacteriocin on Optimised Media and relative concentration dilution 


\section{Results}

\section{Potency of Bacteriocins and its Resistance to Deactivating Factors}

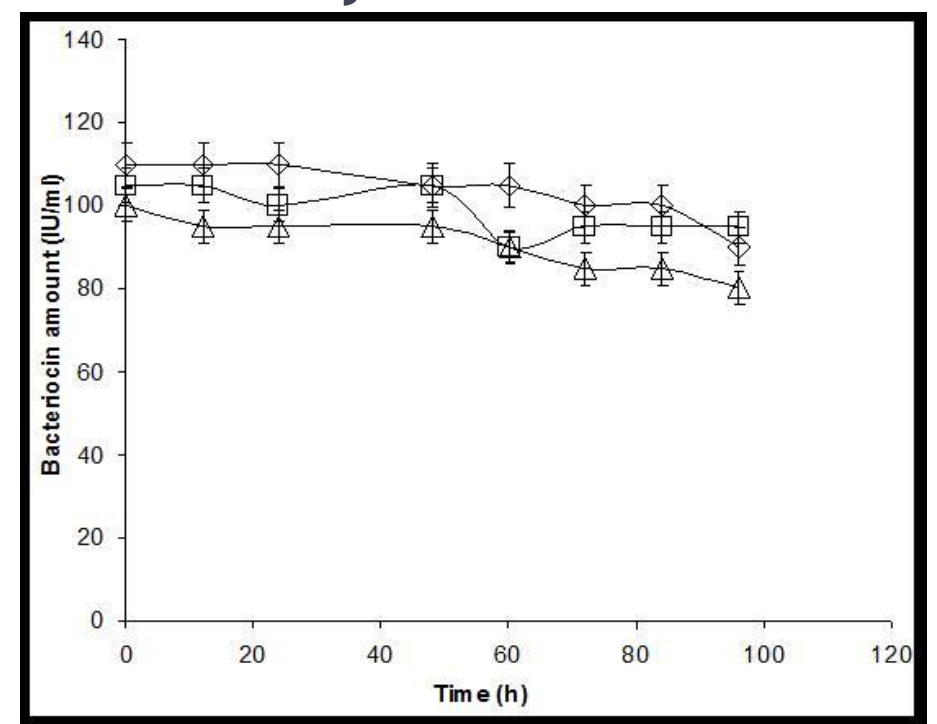

Figure 9 Stability of potency of L.casei bacteriocin up to $96 \mathrm{~h}$ treated with heat $\left(80{ }^{\circ} \mathrm{C}\right)$ $(\diamond)$ treated with microfiltration ( $\square$ ) \&without any treatment $(\Delta)$

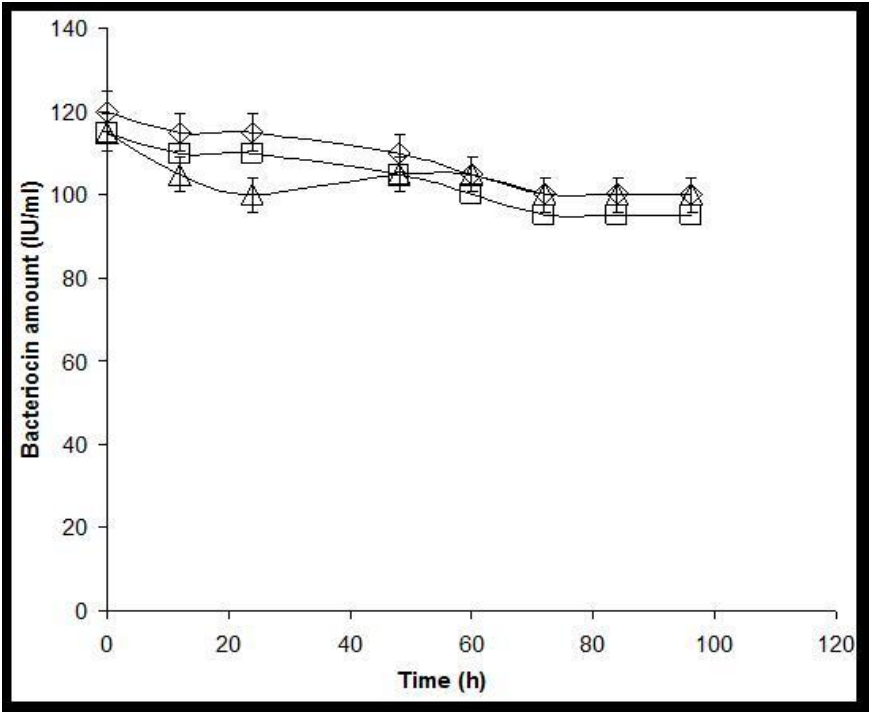

Figure 10 Stability of potency of L.plantartum bacteriocin up to $96 \mathrm{~h}$ treated with heat $\left(80{ }^{\circ} \mathrm{C}\right)$ $(\diamond)$ treated with microfiltration ( $\square$ ) \&without any treatment $(\Delta)$

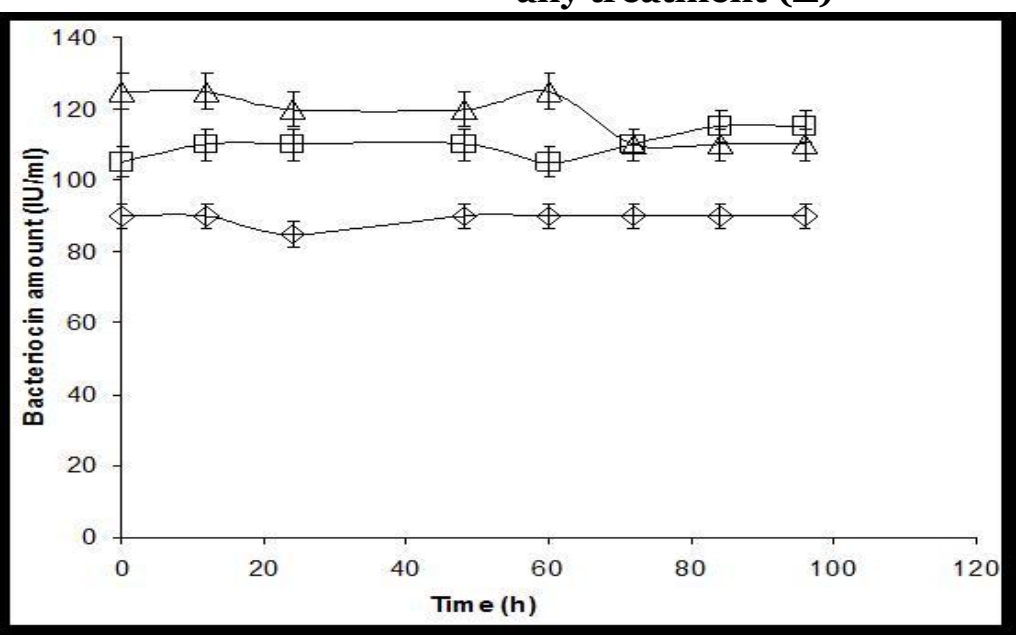

The potency of the bacteriocins was tested under numerous conditions that could possibly deactivate them.

These conditions include heat treatment, storage capacity, filtration and dilution.

It was proven that bacteriocins are relatively stable Figure 11 Stability of potency of L.lactis bacteriocin up to $96 \mathrm{~h}$ treated with heat $\left(80{ }^{\circ} \mathrm{C}\right)$ $(\diamond)$ treated with microfiltration ( $\square$ ) \&without any treatment $(\Delta)$ molecules that

should be commercially

exploited. 


\section{Results}

Stability of the Bacteriocins produced from the selected Lactobacilli

L.delbruckii

\begin{tabular}{|c|c|c|c|c|c|c|c|c|c|}
\hline \multirow{3}{*}{$\begin{array}{l}\text { Lactobacilli } \\
\text { Bacteriocins }\end{array}$} & \multicolumn{9}{|c|}{ Concentrated extracts } \\
\hline & \multicolumn{3}{|l|}{$24 h$} & \multicolumn{3}{|l|}{$48 \mathrm{~h}$} & \multicolumn{3}{|l|}{$72 \mathrm{~h}$} \\
\hline & $\begin{array}{l}\text { Growth } \\
\text { rate } \\
(\mu, h-1)\end{array}$ & $\begin{array}{l}\text { Doubling } \\
\text { time } \\
(t d)\end{array}$ & $\begin{array}{l}\text { Potency } \\
\text { IU/ml }\end{array}$ & $\begin{array}{l}\text { Growth } \\
\text { rate } \\
(\mu, h-1)\end{array}$ & $\begin{array}{l}\text { Doubling } \\
\text { time }(t d)\end{array}$ & $\begin{array}{l}\text { Potency } \\
\text { IU/ml }\end{array}$ & $\begin{array}{l}\text { Growth } \\
\text { rate } \\
(\mu, h-1)\end{array}$ & $\begin{array}{l}\text { Doubling } \\
\text { time }(t d)\end{array}$ & $\begin{array}{l}\text { Potency } \\
\text { IU/ml }\end{array}$ \\
\hline L.casei & 0.15 & 4.60 & 100 & 0.001 & \begin{tabular}{|l|}
$\begin{array}{l}\text { Stationary } \\
\text { phase }\end{array}$ \\
\end{tabular} & 100 & -0.003 & \begin{tabular}{|l} 
No \\
growth
\end{tabular} & 100 \\
\hline L.plantarum & 0.16 & 4.31 & 95 & 0.007 & $\begin{array}{l}\text { Stationary } \\
\text { phase }\end{array}$ & 95 & -0.002 & \begin{tabular}{|l} 
No \\
growth
\end{tabular} & 95 \\
\hline L.lactis & 0.14 & 4.92 & 103 & 0.006 & \begin{tabular}{|l|}
$\begin{array}{l}\text { Stationary } \\
\text { phase }\end{array}$ \\
\end{tabular} & 103 & -0.001 & \begin{tabular}{|l} 
No \\
growth
\end{tabular} & 103 \\
\hline
\end{tabular}

Table 6. Stability of Bacteriocin from Lactobacilli crude extracts 


\section{Results \\ Stability of the Bacteriocins produced from the selected Lactobacilli L.delbruckii}

\begin{tabular}{|c|c|c|c|c|c|c|}
\hline \multirow{3}{*}{$\begin{array}{l}\text { Lactobacilli } \\
\text { Bacteriocins }\end{array}$} & \multicolumn{6}{|c|}{ Concentrated extracts } \\
\hline & \multicolumn{3}{|l|}{$24 h$} & \multicolumn{3}{|l|}{$48 h$} \\
\hline & $\begin{array}{ll}\text { Growth rate } \\
(\mu, h-1)\end{array}$ & $\begin{array}{l}\text { Doubling time } \\
(t d)\end{array}$ & $\begin{array}{l}\text { Potency } \\
\text { IU/ml }\end{array}$ & $\begin{array}{ll}\text { Growth rate } \\
(\mu, h-1)\end{array}$ & $\begin{array}{l}\text { Doubling time } \\
(t d)\end{array}$ & $\begin{array}{l}\text { Potency } \\
\text { IU/ml }\end{array}$ \\
\hline L.casei & 0.07 & 9.85 & 150 & -0.006 & No growth & 150 \\
\hline L.plantarum & 0.06 & 11.5 & 155 & -0.003 & No growth & 155 \\
\hline L.lactis & 0.06 & 11.5 & 155 & -0.002 & No growth & 155 \\
\hline
\end{tabular}

Table 7. Stability of Concentrated Bacteriocin from Lactobacilli crude extracts 


\section{Conclusions and Future Recommendations}

Conclusions

- An optimised growth medium for Lactobacilli and the in vitro production of bacteriocins was developed.

- A novel quantification method of their amount was developed.

- Bacteriocins are proven to be thermostable, resistant to mechanical stress and with strong potency.

Future work

- A mathematical model could be developed forecasting the production of bacteriocins.

- The bacteriocins could be tested in a wider area of target strains.

- Several other nutrient sources could be tested. 


\section{neknouledgments}

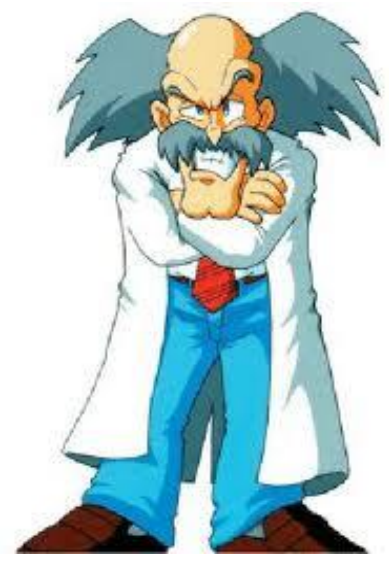

Dr.R.W.Lovitt

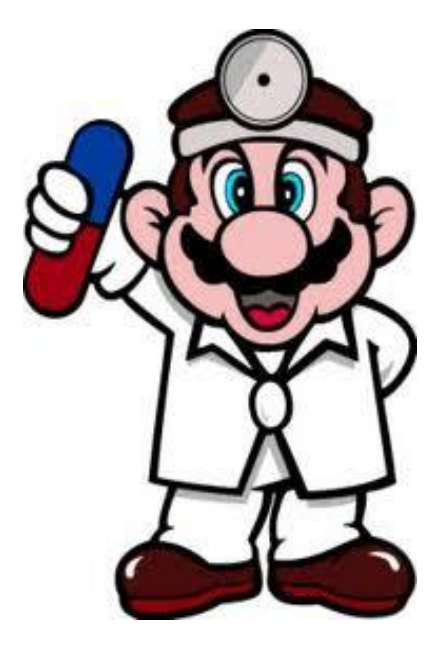

Dr.M.Jones

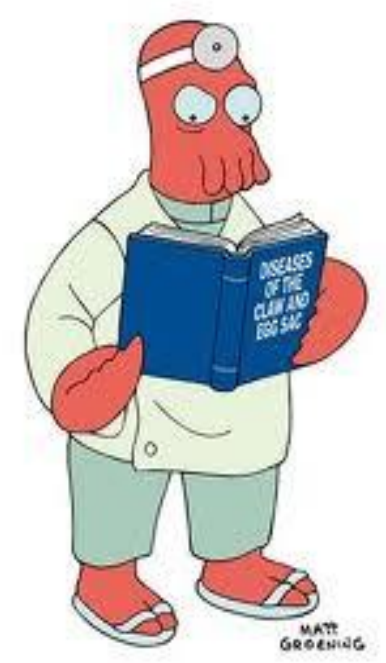

Dr.P.Williams

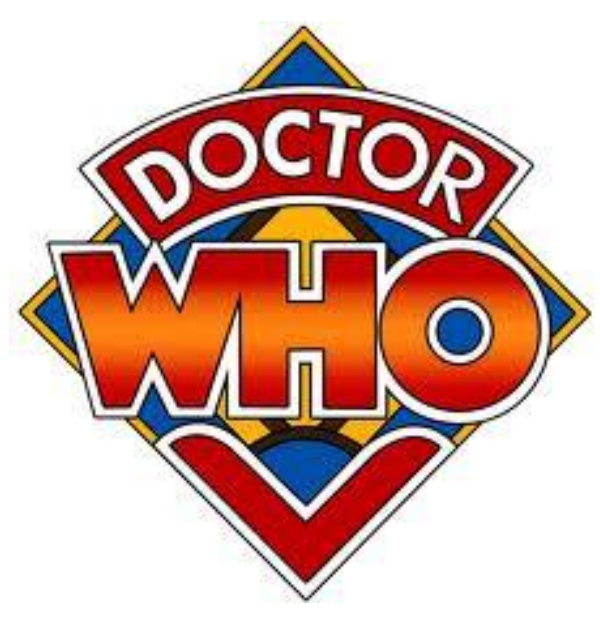

Dr.l.Jung

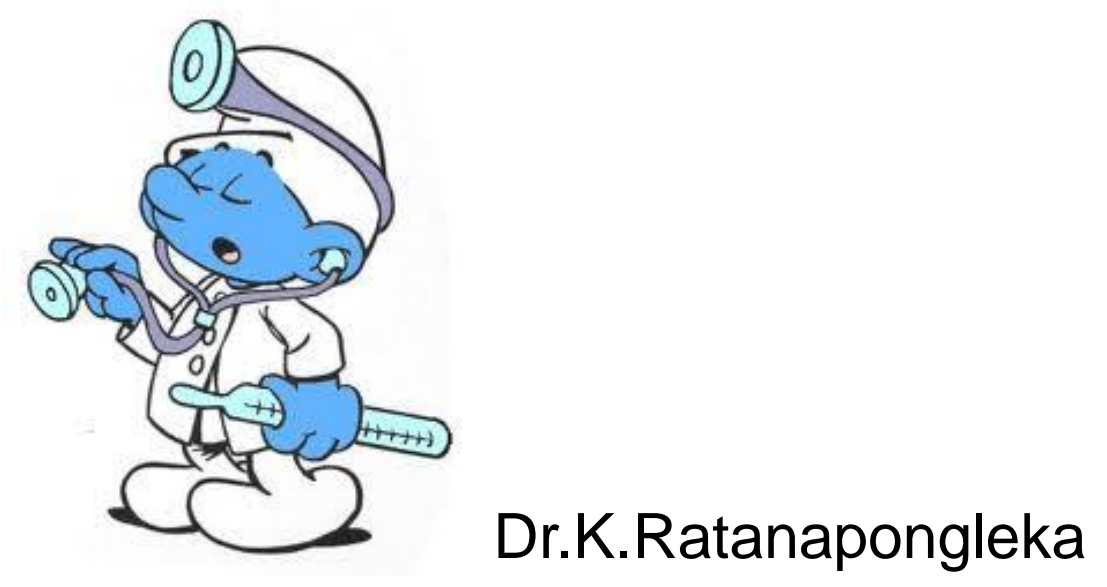




\section{Questions}

Email:

M.Zacharof@swansea.ac.uk

\section{Thank you!!!}

